\title{
A MEMS-Based Active-Head Slider for Flying Height Control in Magnetic Recording*
}

\begin{abstract}
Kenji SUZUKI $^{* *}$ and Masayuki KURITA***
This paper describes design and fabrication of a MEMS-based active-head slider using a PZT thin film for flying height control in hard disk drives. A piezoelectric cantilever integrated in an air bearing slider is used to adjust the flying height individually. A novel air bearing surface (ABS) geometry that minimizes the aerodynamic lift force generated beneath the head has been designed on the basis of the molecular gas film lubrication (MGL) theory. The slider with PZT actuator was fabricated monolithically by silicon micromachining. Performance of the actuator was tested by using an optical surface profiler. Furthermore, the fabricated slider was mounted on a suspension and flying height of the slider above a spinning disk has been measured by means of interferometry. Change in the head-disk spacing has been successfully confirmed by applying voltage to the PZT actuator.
\end{abstract}

Key Words: Microelectromechanical Systems (MEMS), Hard Disk Drive, Air Bearing Surface, Piezoelectric Thin Film, Flying Height, Sol-Gel Method

\section{Introduction}

Air bearing sliders in hard disk drives (HDD) are flying above spinning magnetic disks by aerodynamic lift force. A read/write head is mounted at the trailing edge of the slider and the spacing between the head and the disk is called flying height. The flying height is determined by balance of the loading force given by a suspension and the lift force generated by an air bearing slider. The flying height is decreasing continuously with increasing recoding density and it has reached as small as $10 \mathrm{~nm}$. In order to achieve higher recording density, further reduction of the flying height is necessary. However, it is difficult because of increase in the risk of contact. The flying height is fluctuated by many factors, such as change of environmental temperature and pressure, waviness and vibration of the disk surface, and seeking operation. In particular, static variation due to environmental pressure change comprises a large proportion of the total variation. Furthermore, individual deviation in the flying height due to manufacturing tolerances is also one of the big issues in such low spacing sliders. Therefore, margin for the flying height variation must be taken into account when the nominal flying height

* Received 10th February, 2004 (No. 04-4055)

** Department of Mechanical Systems Engineering, Kogakuin University, 2665-1 Nakano-machi, Hachioji, Tokyo 192-0015, Japan.

E-mail: ksuzuki@cc.kogakuin.ac.jp

*** Mechanical Engineering Research Laboratory, Hitachi, Ltd., 502 Kandatsu, Tsuchiura, Ibaraki 300-0013, Japan. E-mail: m-kurita@rd.hitachi.co.jp is designed.

In this study, an active-head slider, which allows the flying height to be adjusted individually using a microactuator integrated in a slider, is proposed to compensate the flying height variation and to maintain a constant ultralow spacing during the read and write operation. An active slider with an actuator was first proposed by YeackScranton et al. ${ }^{(1)}$ in 1990 for contact recording. Since then, various kinds of active sliders using a bulk $\mathrm{PZT}^{(2)}$, thin film $\mathrm{PZT}^{(3)}$ and thermal actuators ${ }^{(4)}$ have been reported. However they have not been in practical use.

\section{Nomenclature}

$b:$ width of the cantilever [m]

$d_{31}:$ piezoelectric strain constant $[\mathrm{m} / \mathrm{V}]$

$E_{i}$ : Young's modulus $\left[\mathrm{N} / \mathrm{m}^{2}\right]$

$\overline{E I}$ : equivalent bending stiffness of the cantilever $\left[\mathrm{Nm}^{2}\right]$

$f:$ natural frequency of the cantilever $[\mathrm{Hz}]$

$h_{i}:$ thickness [m]

$H$ : dimensionless bearing clearance

$L:$ length of the cantilever [m]

$l:$ length of the slider $[\mathrm{m}]$

$M:$ mass of cantilever end $[\mathrm{kg}]$

$P:$ dimensionless air pressure

$\bar{Q}_{P}$ : relative Poisuille flow rate

$U:$ circumferential disk speed $[\mathrm{m} / \mathrm{s}]$

$V:$ drive voltage of the cantilever [V]

$W$ : loading force of the slider [N]

$w:$ width of the slider [m] 
$X, Y$ : coordinates of the air bearing surface

$\delta:$ displacement of the cantilever tip [m]

$\Lambda$ : bearing number

$\rho_{i}:$ density of silicon and PZT $\left[\mathrm{kg} / \mathrm{m}^{3}\right]$

subscript

$$
\begin{aligned}
& 1: \text { PZT } \\
& 2: \text { silicon }
\end{aligned}
$$

\section{Design and Numerical Analysis}

\subsection{Actuation schemes}

There are two possible actuation schemes for the flying height control: direct actuation and indirect actuation. In the direct actuation scheme, the slider consists of a primary air-bearing slider as a stable reference platform and a secondary low-mass slider to which a read/write head is attached. Two sliders are interconnected by a deformable actuator which drives the head directly. Yeack-Scranton et al. ${ }^{(1)}$ developed an active slider in this scheme by mounting a bulk piezoelectric material in a slider. Kurita et al. ${ }^{(2)}$ and Tagawa et al. ${ }^{(3)}$ has been also studied the direct head actuation using a PZT actuator.

On the other hand, in the indirect actuation scheme, a whole or partial air bearing surface (ABS) is deformed by an actuator integrated in a slider, which results in the change of the flying height indirectly. Khanna et al. ${ }^{(5)}$ proposed a deformable slider using a bulk PZT in 1990. Recently, a partially deformable ABS using a thermal actuator $^{(4)}$ has been proposed.

In this study, the direct actuation scheme is adopted because of low driving force, which is suitable for MEMSbased thin film actuators.

\subsection{Structural design}

A schematic diagram of the newly designed activehead slider is shown in Fig. 1. The slider has a standard "pico-slider" size ( 1 by 1.25 by $0.3 \mathrm{~mm}$ ). A cantilever with a PZT thin film coated on its surface is integrated in the central rear part of the slider and a head is attached to the free end of the cantilever. The cantilever is isolated from the primary slider by two trenches. Figure 2 shows the structure of the piezoelectric cantilever. The PZT layer is sandwiched by two metal electrodes and expands or contracts laterally by applying voltage between two electrodes, which causes the vertical motion of the head. This structure is called the piezoelectric unimorph cantilever.

Dimensions of the cantilever were determined so as to meet following conditions: the displacement of the cantilever is more than $30 \mathrm{~nm}$ and the resonant frequency is more than $100 \mathrm{kHz}$. The thickness of the PZT thin film is restricted by crack formation during the deposition process and determined to be $2 \mu \mathrm{m}$. The displacement of the free end of the cantilever $\delta$ is expressed as:

$$
\delta=\frac{3 m n}{1+4 m n+6 m n^{2}+4 m n^{3}+m^{2} n^{4}} \frac{d_{31} L^{2} V}{h_{1}^{2}}
$$

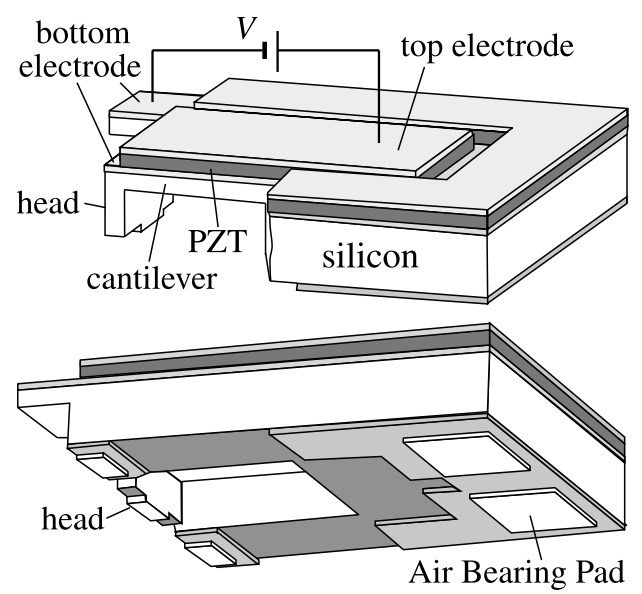

Fig. 1 Schematic diagram of the active head slider

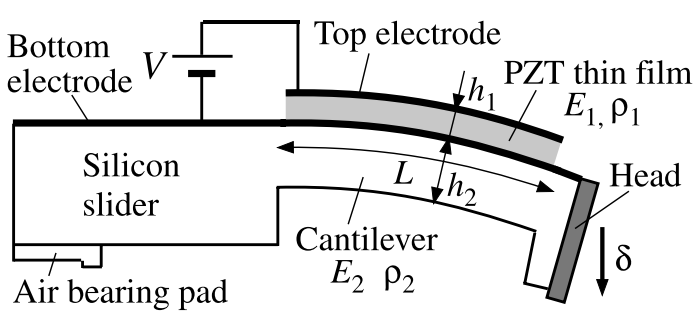

Fig. 2 Structure of piezoelectric unimorph cantilever

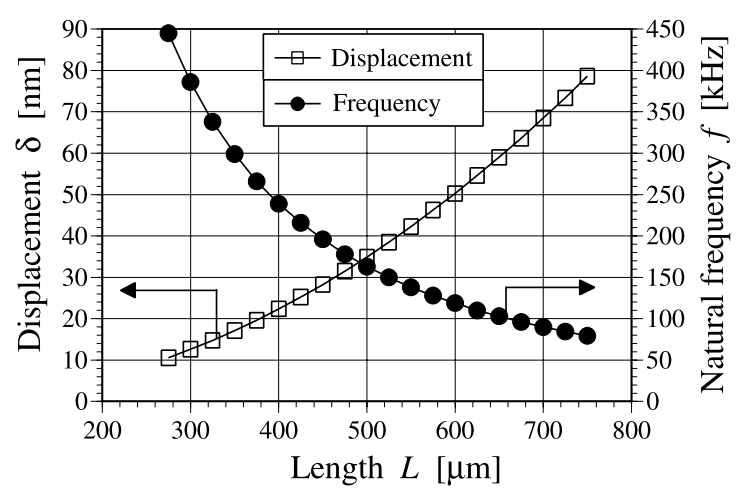

Fig. 3 Displacement and resonant frequency of the cantilever

$$
m=\frac{E_{2}}{E_{1}} \quad, \quad n=\frac{h_{2}}{h_{1}}
$$

Resonant frequency of the cantilever is approximately given by following equations:

$$
\begin{aligned}
& f=\frac{1}{2 \pi L^{2}} \sqrt{\frac{3 \overline{E I}}{M / L+0.236 b\left(\rho_{1} h_{1}+\rho_{2} h_{2}\right)}} \\
& \overline{E I}=\frac{1+4 m n+6 m n^{2}+4 m n^{3}+m^{2} n^{4} \frac{E_{1} b h_{1}^{3}}{12}}{1+m n}
\end{aligned}
$$

The resonant frequency $f$ and the displacement $\delta$ are plotted in Fig. 3 as a function of the cantilever length, based on the following assumptions: $h_{1}=2 \mu \mathrm{m}, h_{2}=$ $50 \mu \mathrm{m}, E_{1}=130 \mathrm{GPa}, E_{2}=63 \mathrm{GPa}, V=5 \mathrm{~V}$ and $d_{31}=$ $-50 \times 10^{-12} \mathrm{~m} / \mathrm{V}$. From the calculations, the length of the cantilever was determined to be $450-650 \mu \mathrm{m}$ range.

\subsection{Air bearing surface (ABS) design}

A novel air bearing surface geometry for the active- 
head slider has been designed based on the molecular gas film lubrication (MGL) theory ${ }^{(6)}$ so as to meet following conditions:

a ) The primary slider always flies at a safe flying height, which is about $20 \mathrm{~nm}$ at the trailing edge, and $90-$ $95 \mathrm{~nm}$ at the leading edge.

b ) The motion of the head has little effect on the flying height and attitude of the primary slider.

In order to meet these conditions, it is necessary to reduce the aerodynamic lift force generated beneath the head. Large aerodynamic force at the cantilever tip causes lift of the whole slider or upward deflection of the cantilever, which counteracts the head motion.

Numerical analysis based on the MGL theory has been carried out to determine the air bearing surface profile. The generalized Reynolds' equation for ultra-thin gas film is expressed as

$$
\frac{\partial}{\partial X}\left(\bar{Q}_{P} P H^{3} \frac{\partial P}{\partial X}\right)+\left(\frac{l}{w}\right)^{2} \frac{\partial}{\partial Y}\left(\bar{Q}_{P} P H^{3} \frac{\partial P}{\partial Y}\right)=\Lambda \frac{\partial(P H)}{\partial X}
$$

The relative Poisuille flow rate $\bar{Q}_{p}$ is introduced to apply the Reynolds' equation to the molecularly thin film. $\bar{Q}_{p}$ is calculated based on the Boltzmann's equation, assuming the accommodation coefficient of the disk and slider surfaces to be $0.9^{(7)}$.

Rectangular unequal spacing meshes were used for the numerical calculation. The control volume method was applied for discretization of the Reynolds' equation including discontinuous bearing clearance. The discretized equation is then linearized with respect to $P$ using the Newton-Raphson method and solved by the column $\operatorname{method}^{(8)}$. The aerodynamic lift force is obtained by integrating the air film pressure $P$ over the air bearing surface. Loading force $W$ and the circumferential speed of the disk $U$ are assumed to be $2.7 \mathrm{gf}$ and $9.61 \mathrm{~m} / \mathrm{sec}$, respectively, and the flying height of the slider was determined by balancing the forces and moments acting on the slider.

Figure 4 shows a newly designed air bearing surface (ABS) profile with five pads. Four of them with $0.1 \mu \mathrm{m}$ deep steps located in each corner generate large aerodynamic pressure and support the load to maintain a stable flying attitude of the slider. On the other hand, the pad at the tip of the cantilever is small and has no step to minimize the aerodynamic lift force.

Figure 5 shows the results of the calculation based on the MGL theory. Pressure distribution for a half width of the ABS is shown. When the cantilever is not deflected $(\delta=0)$, the flying heights of the primary slider and the head at the cantilever tip are both $20 \mathrm{~nm}$, as shown in Fig. 5 (a). When the deflection of $15 \mathrm{~nm}$ is given to the tip of the cantilever, the flying heights of the slider and the head become $20.3 \mathrm{~nm}$ and $5.3 \mathrm{~nm}$, respectively, as shown in Fig. 5 (b). These results indicate that the lift of the pri-

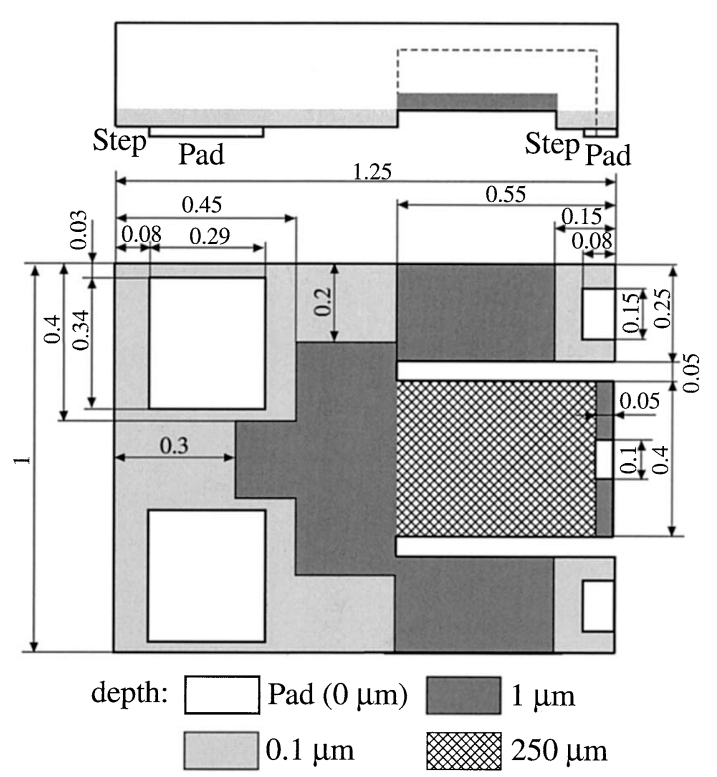

Fig. 4 Air bearing surface profile

(a)

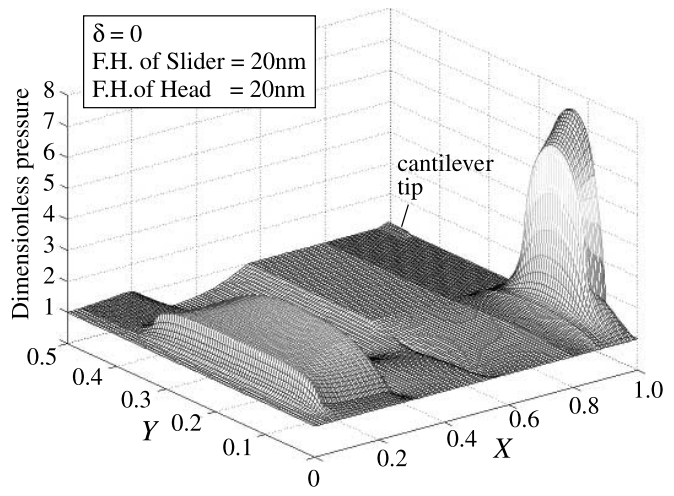

(b)

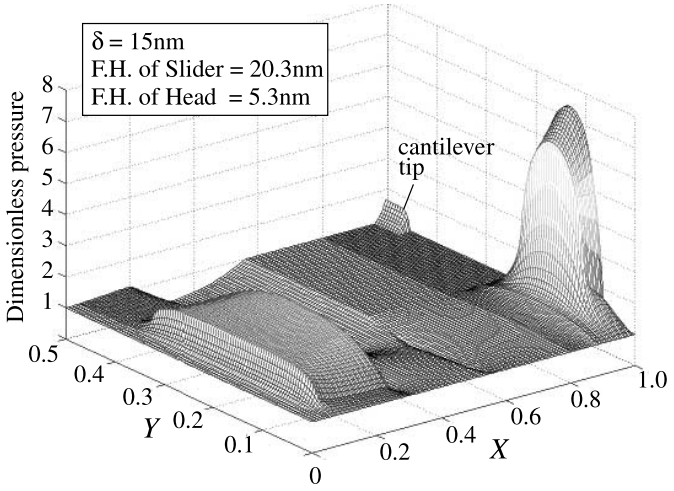

Fig. 5 Pressure distribution

mary slider due to the head motion is only $0.3 \mathrm{~nm}$. The pressure generated at the tip of the cantilever is small even when the head is close to the disk. The aerodynamic lift force at the tip of the cantilever is evaluated to be $14.5 \mathrm{mgf}$, which is $0.5 \%$ of the loading force.

Variation of the flying heights of the slider and the head with applied voltage was also calculated by coupling the MGL calculation with the piezoelectric analysis using 


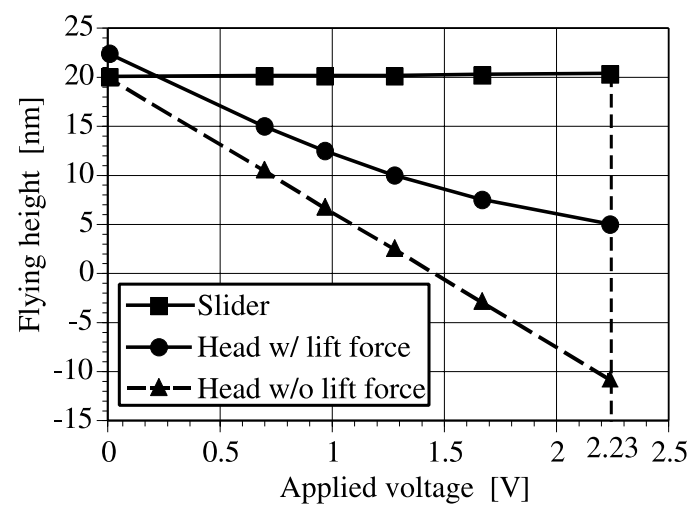

Fig. 6 Variation of flying height with applied voltage

the finite element method. The results of the calculation is shown in Fig. 6. The broken line indicates the deflection of the cantilever tip when the aerodynamic lift force dose not act on the cantilever. The deflection varies linearly with increasing drive voltage. On the other hand, the flying height with aerodynamic lift force (solid line with filled circles) exhibits nonlinear behavior because of the upward deflection of the cantilever due to the lift force. The difference between these two curves indicates the amount of the upward deflection. The primary slider maintains almost constant flying height regardless of the deflection of the cantilever. The voltage required to lower the head from $20 \mathrm{~nm}$ to $5 \mathrm{~nm}$ is evaluated to be $2.23 \mathrm{~V}$.

\section{Fabrication}

The active-head sliders with a PZT unimorph cantilevers have been fabricated monolithically by silicon micromachining process. MEMS technology allows hundreds of sliders with piezoelectric cantilevers to be fabricated simultaneously without assembly process. Figure 7 shows the fabrication process. First, a 300- $\mu$ m-thick silicon wafer is etched from the backside by $\mathrm{KOH}$ solution to form the $0.1-\mu \mathrm{m}$-deep steps and $1-\mu \mathrm{m}$-deep recesses for the air bearing surface. Then the wafer is thermally oxidized to deposit $0.1-\mu \mathrm{m}$-thick insulating layer. After that, a bottom electrode (Pt/Ti), a PZT film, and a top electrode $(\mathrm{Au} / \mathrm{Cr})$ are deposited sequentially. The PZT film is coated by sol-gel method. A 2 - $\mu$ m-thick crack-free PZT film was successfully deposited by multiple spin coating and heat treatment. The top electrode and the PZT film are then patterned by ECR (electron cyclotron resonance) etching. Subsequently, the bottom electrode and the upper part of the silicon slider are patterned by ECR etching and deep-RIE (reactive ion etching), respectively. Lastly, the 50- $\mu \mathrm{m}$-thick cantilevers and the sidewalls of the sliders are formed simultaneously by deep-RIE and the sliders are separated individually from the wafer.

Figure 8 shows SEM micrographs of the fabricated sliders. The $250-\mu \mathrm{m}$-deep perpendicular walls are etched successfully.
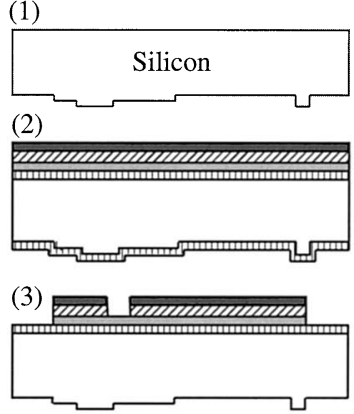

Fig. 7 Fabrication process
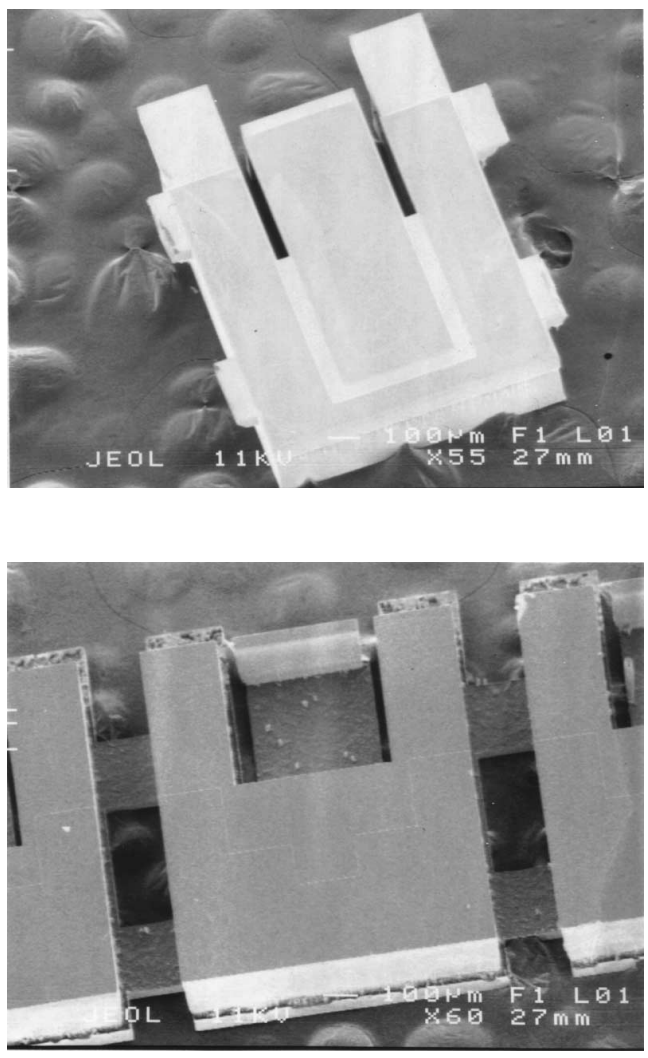

Fig. 8 SEM micrographs of the slider

\section{Testing and Results}

The displacement of the cantilever tip measured by an optical surface profiler is plotted as a function of applied voltage in Fig. 9. When the voltage from $-18 \mathrm{~V}$ to $+15 \mathrm{~V}$ is applied, the hysteresis of the displacement is observed, as shown in Fig. 9 (a). However the displacement in the low voltage range from $-5 \mathrm{~V}$ to $5 \mathrm{~V}$ is almost proportional to the applied voltage and the effect of the hysteresis becomes small, as shown in Fig. 9(b), because inversion of polarization in the PZT film does not occur in the low voltage range. When the voltage of $5 \mathrm{~V}$ is applied, displacement of more than $60 \mathrm{~nm}$ is obtained at the tip of the $550 \mu \mathrm{m}$-long cantilever, which is large enough to control the flying height. Piezoelectric strain constant $d_{31}$ is estimated as $182 \times 10^{-12} \mathrm{~m} / \mathrm{V}$ from Eq. (1). 
(a)

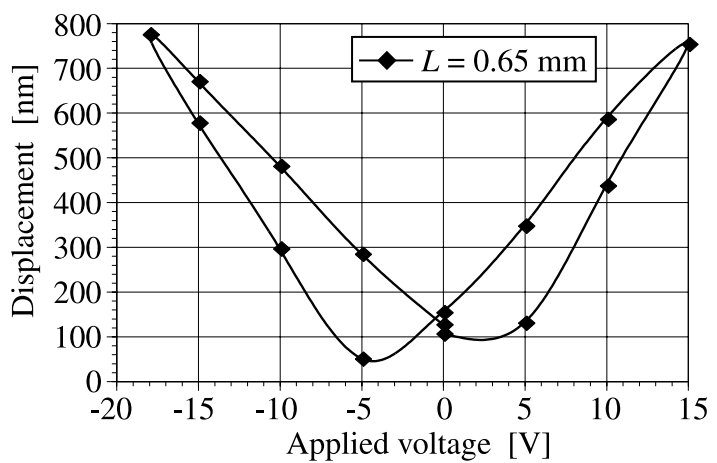

(b)

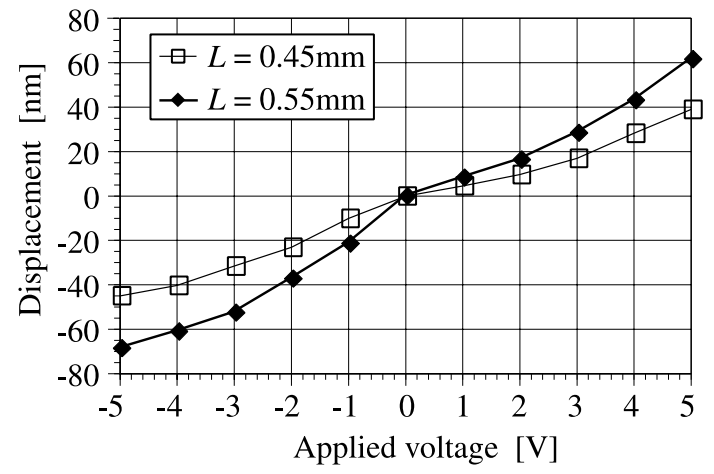

Fig. 9 Displacement of the cantilever tip, (a) $-18 \mathrm{~V}$ to $+15 \mathrm{~V}$, (b) $-5 \mathrm{~V}$ to $+5 \mathrm{~V}$

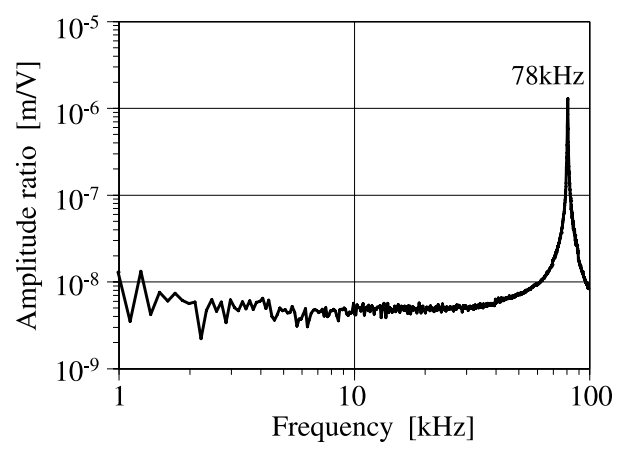

Fig. 10 Frequency response of the cantilever

The frequency response of the cantilever was also tested using laser Doppler vibrometer. The result for a $650-\mu \mathrm{m}$-long and $40-\mu \mathrm{m}$-thick cantilever is shown in Fig. 10. The resonant frequency was $78 \mathrm{kHz}$ and the $Q$ value was estimated approximately to be 250 . The resonant frequency of $550 \mu \mathrm{m}$-long cantilever was found to be over $100 \mathrm{kHz}$.

The fabricated slider was then mounted on a suspension by glue, as shown in Fig. 11, and the top and bottom electrodes were connected electrically to contact pads on the suspension using solder balls of $100 \mu \mathrm{m}$ in diameter. The slider was loaded and levitated above a spinning disk and the flying height was measured by means of interferometry through a transparent glass disk, as shown in Fig. 12. The circumferential speed of the disk beneath the slider was $9.6 \mathrm{~m} / \mathrm{s}$.

The results of the flying height measurements are solder ball
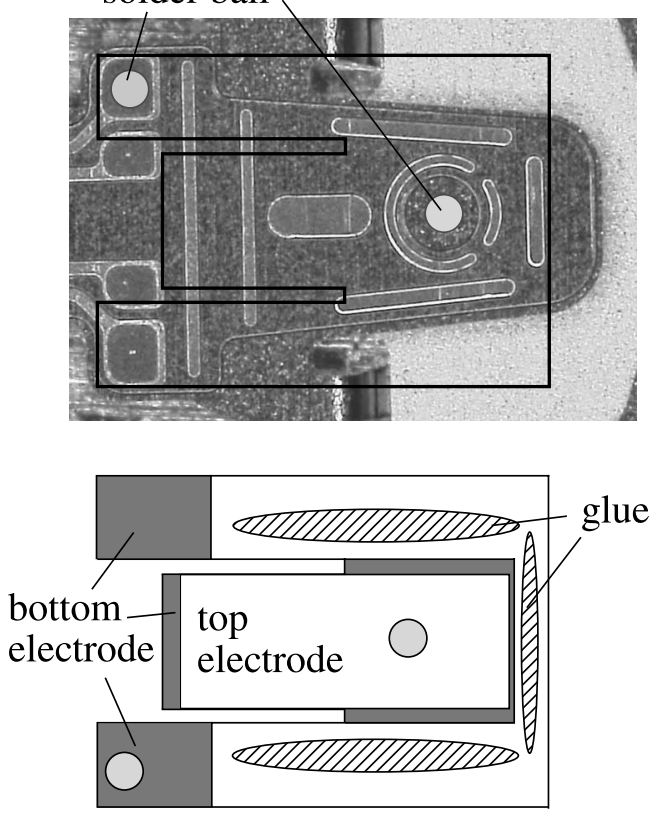

Fig. 11 Mounting on a suspension

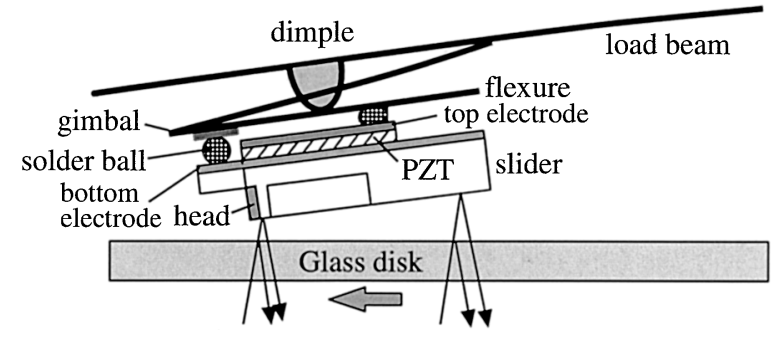

Fig. 12 Measurement of flying height through a transparent glass disk

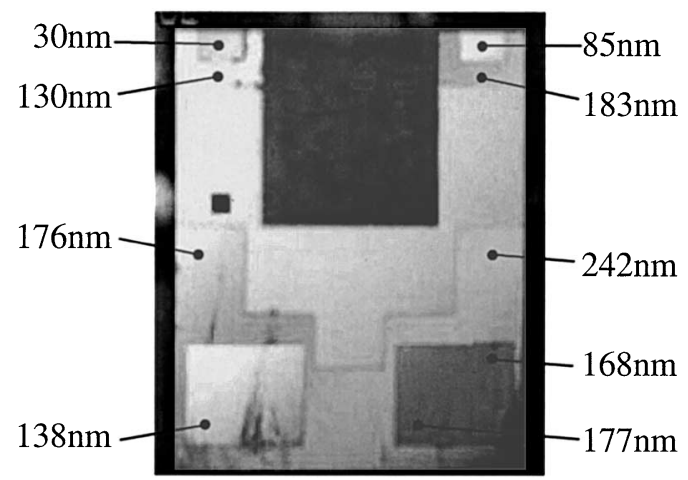

Fig. 13 Flying height of the slider

shown in Fig. 13. The slider maintained almost constant flying height above a spinning disk although it was tilted. The minimum flying height was found to be $30 \mathrm{~nm}$ at the left trailing edge of the slider. On the other hand, the flying height of the cantilever tip could not be measured quantitatively because the cantilever was warped upward by residual stress. The deflection of the cantilever tip was about $300 \mathrm{~nm}$. Instead, shift of the interference fringes was observed on the pad at the cantilever tip by changing 

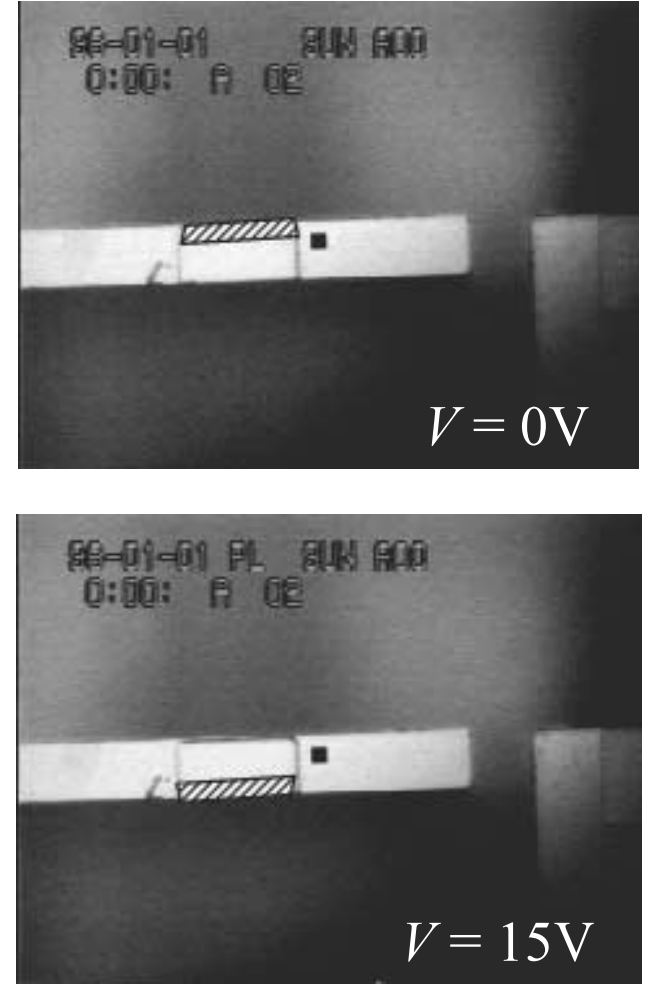

Fig. 14 Shift of the interference fringes on the pad at the cantilever tip. The hatched areas show a red interference band.

the drive voltage stepwise from 0 to $15 \mathrm{~V}$. Hatched areas in Fig. 14 indicate a red interference band observed on the pad. The results demonstrate that the flying height of the cantilever tip can be adjusted using a PZT thin film.

The stress control of the cantilever is key issues to realize the active head slider. Modification of the materials, thickness, and anealing process of the multilayered cantilever may be effective to reduce the residual stress. The tilt of the slider can be reduced by improving the accuracy of the assembly process.

\section{Conclusion}

An active-head slider for adjusting the flying height individually using a piezoelectric cantilever integrated in a slider has been proposed. This slider allows the margin for the flying height variation due to manufacturing tolerances and environmental changes to be eliminated and an ultralow flying height can be realized with high reliability.

The structure of a piezoelectric unimorph cantilever and a 5-pad air bearing surface which minimizes the aerodynamic lift force beneath the head have been designed on the basis of the MGL theory. The relationship between deflection of the piezoelectric cantilever and applied voltage has been predicted theoretically by coupling the MGL theory with the piezoelectric analysis.

A fabrication process of the active-head slider has been developed using MEMS technology including sol- gel PZT coating and bulk micromachining by deep RIE.

Performance of the actuator was tested by using an optical surface profiler. Displacement of more than $60 \mathrm{~nm}$ was obtained at the cantilever tip with drive voltage of 5 volts. Furthermore, the flying height measurement above a spinning disk was carried out. Vertical displacement of the cantilever tip was successfully confirmed while the slider was flying stably above a disk. The experimental results demonstrate the possibility of controlling flying height using a PZT thin film.

\section{Acknowledgments}

The authors would like to thank Prof. I. Shimoyama, The University of Tokyo, and Dr. R. Maeda, Advanced Institute of Science and Technology, for the provision of laboratory facilities and technical support for microfabrication.

\section{References}

( 1 ) Yeack-Scranton, C.E., Khanna, V.D., Etzold, K.F. and Praino, A.P., An Active Slider for Practical Contact Recording, IEEE Trans. on Magnetics, Vol.26, No.5 (1990), pp.2478-2483.

( 2 ) Kurita, M., Yamazaki, T., Kohira, H., Matsumoto, M., Tsuchiyama, R., Xu, J., Harada, T., Inoue, Y., Su, L. and Kato, K., An Active-Head Slider with a Piezoelectric Actuator for Controlling Flying Height, IEEE Trans. on Magnetics, Vol.38, No.5 (2002), pp.21022104.

( 3 ) Tagawa, N., Design and Fabrication of MEMS-Based Active Sliders Using Double-Layered Composite PZT Thin Films in Hard Disk Drives, Digest of the Asia Pacific Magnetic Recording Conference 2002, (2002), pp.CP4-01-02.

(4) Machtle, P., Berger, R., Dietzel, A., Despont, M., Haberle, W., Stutz, R., Binnig, G.K. and Vettiger, P., Integrated Microheaters for In-Situ Flying-Height Control of Sliders Used in Hard-Disk Drives, IEEE Proceedings of MEMS Conference 2001, (2001), pp.196199.

( 5 ) Khanna, V.D. and Hendriks, F., Programmable Air Bearing Sliders for Magnetic Storage, STLE Special Publication, SP-29, (1990), pp.43-48.

( 6 ) Fukui, S. and Kaneko, R., Analysis of Ultra-Thin Gas Film Lubrication Based on Linearized Boltzmann Equation, First Report: Derivation of a Generalized Lubrication Equation Including Thermal Creep Flow, Trans. ASME, J. Tribology, Vol.110 (1988), pp.253261.

( 7 ) Fukui, S. and Kaneko, R., A Database for Interpolation of Poiseuille Flow Rates for High Knudsen Number Lubrication Problems, Trans. ASME, J. Tribology, Vol.112 (1990), pp.78-83.

( 8 ) Castelli, V. and Pirvics, J., Review of Numerical Methods in Gas Bearing Film Analysis, Trans. ASME, J. Lubrication Technology, (1990), pp.777-792. 